\title{
Global existence and blow-up of solution for the semilinear wave equation with interior and boundary source terms
}

\author{
Hongwei Zhang $^{1 *}$ (D), Wenxiu Zhang ${ }^{1}$ and Qingying $\mathrm{Hu}^{1}$
}

"Correspondence:

whz661@163.com

'Department of Mathematics,

Henan University of Technology,

Zhengzhou, P.R. China

\section{每 Springer}

\begin{abstract}
This paper is concerned with semilinear wave equations with nonlinear interior and boundary sources and subject to a nonlinear dynamical boundary condition. By using the potential well method combined with a standard continuous argument, under appropriate assumptions imposed on the source term, we establish global existence of solutions. Moreover, for certain initial data in the unstable set, the finite time blow-up phenomenon is exhibited.
\end{abstract}

MSC: 35A01; 35L35; 35B44; 76X05

Keywords: Global existence; Blow-up; Dynamical boundary condition; Wave equation; Potential well theory

\section{Introduction}

In this paper, we consider the following model of semilinear wave equation with nonlinear interior and boundary sources:

$$
\begin{aligned}
& u_{t t}-\Delta u_{t t}-\Delta u=g(u), \quad t>0, \\
& \frac{\partial u_{t t}}{\partial v}+\frac{\partial u}{\partial v}+u=f(u), \quad x \in \Gamma, t>0, \\
& u(x, 0)=u_{0}(x), \quad u_{t}(x, 0)=u_{1}(x), \quad x \in \Omega,
\end{aligned}
$$

in a bounded domain $\Omega \subset R^{n}$ with smooth boundary $\partial \Omega=\Gamma$, where $f(s), g(s)$ are continuous functions and $\frac{\partial}{\partial \nu}$ denotes the unit outer normal derivative. In this paper, we take $f(s)=b|u|^{k-1} u, g(s)=a|u|^{p-1} u$, where $a, b$ are positive constants. For simplicity, we take $a=b=1$.

Problem (1.1)-(1.3) arises from a model equation of ion-sound waves in 'non-magnetized' plasma taking account of nonlinear sources localized on the boundary [1-6]. This generates a nonlinear dynamical boundary condition which is 'close' to the nonlinear Neumann-Dirichlet condition [1]. Problem (1.1)-(1.3) when $g(u)=0$ (i.e., without interior source term) has been considered by Korpusov [1]. Korpusov proved that the solution of problem (1.1)-(1.3) exists locally in time for all initial data $u_{0}(x), u_{1}(x) \in H^{1}(\Omega)$ and that the solution blows up in a finite time provided that the initial data (the functions $u_{0}(x)$ and

(c) The Author(s) 2019. This article is distributed under the terms of the Creative Commons Attribution 4.0 International License (http://creativecommons.org/licenses/by/4.0/), which permits unrestricted use, distribution, and reproduction in any medium, provided you give appropriate credit to the original author(s) and the source, provide a link to the Creative Commons license, and indicate if changes were made. 
$\left.u_{1}(x)\right)$ are sufficiently 'large', i.e.,

$$
\begin{aligned}
& \int_{\Omega}\left[\left|\nabla u_{1}\right|^{2}+\left|u_{1}\right|^{2}\right] d x+\int_{\Omega}\left|\nabla u_{0}\right|^{2} d x<2 \int_{\Gamma} \int_{0}^{u_{0}} f(s) d s d x, \\
& \int_{\Omega}\left[\nabla u_{1} \nabla u_{0}+u_{0} u_{1}\right] d x>0
\end{aligned}
$$

by using a modification of Levine's energy method [7, 8]. Park and Kim [9] discussed the existence and uniform decay rates of the energy of solutions for the following problem:

$$
\begin{aligned}
& \left|u_{t}\right|^{\rho} u_{t t}-\beta \Delta u_{t t}-\Delta u-\Delta u_{t}=0, \quad \text { in } \Omega \times(0, \infty), \\
& \beta \frac{\partial u_{t t}}{\partial v}+\frac{\partial u}{\partial v}+\frac{\partial u_{t}}{\partial v}+u=\int_{0}^{t} g(t-s)\left|u_{t}(s)\right|^{\gamma} u_{t}(s) d s, \quad \text { on } \Gamma_{1} \times(0, \infty), \\
& u=0, \quad \text { on } \Gamma_{0} \times(0, \infty),
\end{aligned}
$$

with initial condition (1.3), where $\Gamma_{1} \cup \Gamma_{0}=\partial \Omega=\Gamma$ and $\Gamma_{1} \cap \Gamma_{0}=\emptyset$. However, as far as we know, until now there have not been many works on this class of problems. In this paper, we will extend the result in [1] for negative initial energy to the semilinear wave equation with positive initial energy and nonlinear interior and boundary sources. It is well known that the presence of the boundary source term in equation (1.2) brings great difficulty due to the fact that the Lopatinskii condition [10] does not hold [11]. A combination of interior and boundary sources with positive initial energy is a much more challenging problem.

To motivate our work, let us recall some results of the following wave equations:

$$
\begin{array}{ll}
u_{t t}-\Delta u+g\left(u_{t}\right)=f(u), & \text { in } \Omega \times(0, T), \\
\frac{\partial u}{\partial v}+u+g_{1}\left(u_{t}\right)=h(u), & \text { on } \Gamma \times(0, T) .
\end{array}
$$

This problem has been widely studied. Several results have been established. It is worth noting the pioneering work of Lasiecka and Tataru [12] in which (1.4) with $g=0$ was conducted under a very weak geometrical condition on $\partial \Omega$. They established the uniform decay rates for the solutions. Vitillaro [13] obtained a full analysis of local and global existence of problem (1.4), (1.5) and (1.3). Recently, Boicu and Lasiecka et al. [11, 14-28] studied problem (1.4), (1.5) and (1.3) with interior and boundary sources and damping terms. They obtained global existence of a unique weak solution and established explicit uniform energy rates. As for blow-up of solutions, they established blow-up results with up-to-critical boundary sources. Especially, at the super-critical level for both interior and boundary sources terms, the blow-up theorem was presented in [15] for initial data of negative energy, and Bociu, Rammaha, and Toundykov [18] proved a blow-up result for weak solutions with nonnegative initial energy.

It is important to observe that similar equations to the one given in (1.1) arise also in the study of viscoelastic plates. Ji and Lasiecka [29] proved that a semilinear Kirchhoff equation

$$
u_{t t}-\Delta u_{t t}+\Delta^{2} u=f(u)
$$

with nonlinear dissipation acting via moments only is uniform energy decay. 
Motivated by these papers, in this paper we aim to investigate the existence and nonexistence of global solutions for problem (1.1)-(1.3) with nonnegative initial energy. More precisely, under appropriate assumptions imposed on the source term, we shall establish global existence of solutions by using the potential well method combined with a standard continuous argument. Moreover, for certain initial data in the unstable set, we will extend the finite time blow-up result in [1] for negative initial energy to the semilinear wave equation with positive initial energy and nonlinear interior and boundary sources. Combining this method with the method of $[18,19,27]$, we can also consider the equation

$$
\begin{aligned}
& u_{t t}-\Delta u_{t t}-\Delta u+\int_{0}^{t} k(t-s) \Delta u(s) d s+h\left(u_{t}\right)=g(u), \\
& \frac{\partial u_{t t}}{\partial v}+\frac{\partial u}{\partial v}+u+h_{1}\left(u_{t}\right)=f(u), \quad x \in \Gamma,
\end{aligned}
$$

with initial condition (1.3). The plan of this article is as follows. In Sect. 2, we introduce some notations, assumptions, and preliminaries. In Sect. 3, we show the main results of this article.

\section{Preliminaries}

In this section, we present some materials needed in the proof of our results. We use the standard Lebesgue space $L^{p}(\Omega)(1<p<\infty)$ and the Sobolev space $H^{1}(\Omega)$ with their usual scalar products and norms. For simplicity, $\|u\|_{L^{p}(\Omega)}=\|u\|_{p}$ and $\|u\|_{L^{q}(\Gamma)}=\|u\|_{q, \Gamma}$ for $1 \leq$ $p, q \leq \infty$. In particular, we denote $\|u\|_{L^{2}(\Omega)}=\|u\|$ and $\|u\|_{L^{2}(\Gamma)}=\|u\|_{\Gamma}$. It is well known that the norm $\left(\|\nabla u\|^{2}+\|u\|_{\Gamma}^{2}\right)^{\frac{1}{2}}$ is equivalent to the norm $\|u\|_{H^{1}(\Omega)}$ on the space $H^{1}(\Omega)$. Thus we put $\|u\|_{H^{1}(\Omega)}=\|u\|_{1, \Omega}=\left(\|\nabla u\|^{2}+\|u\|_{\Gamma}^{2}\right)^{\frac{1}{2}}$ for $u \in H^{1}(\Omega)$. The constants $C$ used throughout this paper are positive generic constants, which may be different in various occurrences.

We assume that

$$
1 \leq p \leq \frac{n}{n-2}, \quad 1 \leq k \leq \frac{n-1}{n-2} \quad \text { if } n \geq 3 ; \quad p \geq 1, \quad k \geq 1 \quad \text { if } n=1,2 .
$$

In this case, we have the Sobolev embedding $H^{1}(\Omega) \hookrightarrow L^{p+1}(\Omega)$ and the Trace-Sobolev embedding $H^{1}(\Omega) \hookrightarrow L^{k+1}(\Gamma)$. In these cases, the embedding constants are denoted by $c_{*}, B_{*}$ respectively, i.e.,

$$
\|u\|_{p+1} \leq c_{*}\|u\|_{1, \Omega}, \quad\|u\|_{k+1, \Gamma} \leq B_{*}\|u\|_{1, \Omega} .
$$

A function $u(x, t)$ of class $L^{\infty}\left(0, T ; H^{1}(\Omega)\right)$ with $u_{t}, u_{t t} \in L^{\infty}\left(0, T ; H^{1}(\Omega)\right)$ is called a weak generalized solution of problem (1.1)-(1.3) [1] if it satisfies the equation

$$
\left(u_{t t}, \phi\right)+\left(\nabla u_{t t}, \nabla \phi\right)+(\nabla u, \nabla \phi)+\int_{\Gamma} u \phi d x=\int_{\Omega}|u|^{p-1} u \phi d x+\int_{\Gamma}|u|^{k-1} u \phi d x
$$

for any $\phi \in H^{1}(\Omega)$ and for almost all $t \in[0, T]$ and the initial condition

$$
u(x, 0)=u_{0}(x), \quad u_{t}(x, 0)=u_{1}(x) .
$$


Theorem 2.1 Let $u_{0}, u_{1} \in H^{1}$ and $p$, $k$ satisfy (2.1), then problem (1.1)-(1.3) has a unique weak generalized solution on $\left[0, T_{0}\right)$ for some $T_{0}>0$, and we have either $T_{0}=+\infty$ or $T_{0}<$ $+\infty$ and

$$
\lim _{t \rightarrow T_{0}} \sup \left[\|u\|_{1, \Omega}^{2}+\left\|u_{t}\right\|_{1, \Omega}^{2}\right]=+\infty
$$

Remark This theorem can be easily established by combining the argument of [30] and Theorem 2.2 in [1], so we omit it.

We define the functional that plays as the "potential energy"

$$
\begin{aligned}
J(u) & =\frac{1}{2}\|\nabla u\|^{2}+\frac{1}{2}\|u\|_{\Gamma}^{2}-\frac{1}{p+1}\|u\|_{p+1}^{p+1}-\frac{1}{k+1}\|u\|_{k+1, \Gamma}^{k+1} \\
& =\frac{1}{2}\|u\|_{1, \Omega}^{2}-\frac{1}{p+1}\|u\|_{p+1}^{p+1}-\frac{1}{k+1}\|u\|_{k+1, \Gamma}^{k+1},
\end{aligned}
$$

and the Nehari functional

$$
I(u)=\|u\|_{1, \Omega}^{2}-\|u\|_{p+1}^{p+1}-\|u\|_{k+1, \Gamma}^{k+1} .
$$

We have also the following energy identity:

$$
E(t)=\frac{1}{2}\left\|u_{t}\right\|_{1, \Omega}^{2}+J(u)=E(0)
$$

In the sequel, a crucial role is played by the Nehari manifold to $I$, that is,

$$
N=\left\{u \in H^{1}(\Omega) \mid I(u)=0,\|u\|_{1, \Omega} \neq 0\right\}
$$

and we can readily give the mountain-pass level $d$ by $d=\inf _{u \in N} J(u)$.

Next, we show some properties related to functions $J(u)$ and $I(u)$ in the following lemma.

Lemma 2.2 Let $u \in H^{1}(\Omega)$ and $\|u\|_{1, \Omega} \neq 0$, then

(i) $\lim _{\lambda \rightarrow 0} J(\lambda u)=0, \lim _{\lambda \rightarrow+\infty} J(\lambda u)=-\infty$;

(ii) There exists unique $\lambda_{0}=\lambda_{0}(u)$ such that $\left.\frac{d}{d \lambda} J(\lambda u)\right|_{\lambda=\lambda_{0}}=0$;

(iii) $J(\lambda u)$ is increasing on $0<\lambda \leq \lambda_{0}$, decreasing on $\lambda_{0} \leq \lambda<+\infty$, and takes the maximum at $\lambda=\lambda_{0}$;

(iv) $I(\lambda u)>0$ for $0<\lambda<\lambda_{0} ; I(\lambda u)<0$ for $\lambda>\lambda_{0}$, and $I\left(\lambda_{0} u\right)=0$.

Proof The first conclusion follows from

$$
J(\lambda u)=\frac{\lambda^{2}}{2}\|u\|_{1, \Omega}^{2}-\frac{\lambda^{p+1}}{p+1}\|u\|_{p+1}^{p+1}-\frac{\lambda^{k+1}}{k+1}\|u\|_{k+1, \Gamma}^{k+1}
$$

As in [19], let $\lambda_{0}$ be the first positive zero of the function $F^{\prime}(x)$ where

$$
F(x)=\frac{1}{2} x^{2}-\frac{c_{*}^{p+1}}{p+1} x^{p+1}-\frac{B_{*}^{k+1}}{k+1} x^{k+1},
$$


and we can verify that the function $F(x)$ is increasing in $0<\lambda \leq \lambda_{0}$, decreasing in $\lambda_{0} \leq \lambda<$ $+\infty$, and $F$ has a maximum at $\lambda=\lambda_{0}$. Then we have that (ii) and (iii) hold. The conclusion (iv) follows from (iii) and the fact that $I(\lambda u)=\lambda \frac{J(\lambda u)}{d \lambda}$.

Lemma 2.3 Let $I(u)$ be the Nehari functional defined in (2.4) and $\lambda_{0}$ be the first positive zero of the function $F^{\prime}(x)$, then (i) if $0<\|u\|_{1, \Omega}<\lambda_{0}$, then $I(u)>0$; (ii) if $I(u)<0$, then $\|u\|_{1, \Omega}>\lambda_{0}$; (iii) if $I(u)=0$ and $\|u\|_{1, \Omega} \neq 0$, i.e., $u \in N$, then $\|u\|_{1, \Omega} \geq \lambda_{0}$.

Proof We note that

$$
1=c_{*}^{p+1} \lambda_{0}^{p-1}+B_{*}^{k+1} \lambda_{0}^{k-1},
$$

so we denote $\phi(x)=c_{*}^{p+1} x^{p-1}+B_{*}^{k+1} x^{k-1}$, then $\phi\left(\lambda_{0}\right)=1$.

(i) Since $\phi(x)$ is a strictly increasing function in $\left(0, \lambda_{0}\right)$, from $0<\|u\|_{1, \Omega}<\lambda_{0}$, we get $\phi\left(\|u\|_{1, \Omega}\right)<\phi\left(\lambda_{0}\right)$ and hence

$$
\begin{aligned}
I(u) & =\|u\|_{1, \Omega}^{2}-\|u\|_{p+1}^{p+1}-\|u\|_{k+1, \Gamma}^{k+1} \\
& \geq\|u\|_{1, \Omega}^{2}\left(1-c_{*}^{p+1}\|u\|_{1, \Omega}^{p-1}-B_{*}^{k+1}\|u\|_{1, \Omega}^{k-1}\right) \\
& =\|u\|_{1, \Omega}^{2}\left(1-\phi\left(\|u\|_{1, \Omega}\right)\right)>0 .
\end{aligned}
$$

(ii) Condition $I(u)<0$ gives

$$
\begin{aligned}
\phi\left(\lambda_{0}\right)\|u\|_{1, \Omega}^{2} & =\|u\|_{1, \Omega}^{2}<\|u\|_{p+1}^{p+1}+\|u\|_{k+1, \Gamma}^{k+1} \\
& \leq\left(c_{*}^{p+1}\|u\|_{1, \Omega}^{p-1}+B_{*}^{k+1}\|u\|_{1, \Omega}^{k-1}\right)\|u\|_{1, \Omega}^{2}=\phi\left(\|u\|_{1, \Omega}\right)\|u\|_{1, \Omega}^{2},
\end{aligned}
$$

which implies $\|u\|_{1, \Omega} \neq 0$ and $\|u\|_{1, \Omega}>\lambda_{0}$ by the monotonicity of $\phi$.

(iii) If $I(u)=0$ and $\|u\|_{1, \Omega} \neq 0$, then

$$
\phi\left(\lambda_{0}\right)\|u\|_{1, \Omega}^{2}=\|u\|_{1, \Omega}^{2}=\|u\|_{p+1}^{p+1}+\|u\|_{k+1, \Gamma}^{k+1} \leq \phi\left(\|u\|_{1, \Omega}\right)\|u\|_{1, \Omega}^{2},
$$

and from the monotonicity of $\phi$ we get $\|u\|_{1, \Omega}>\lambda_{0}$.

Lemma 2.4 Let $I(u)$ be the Nehari functional defined in (2.4), then (i) $d \geq d_{0}=\left(\frac{1}{2}-\right.$ $\left.\max \left\{\frac{1}{p+1}, \frac{1}{k+1}\right\}\right) \lambda_{0}^{2}$; (ii) if $u \in H^{1}$ and $I(u)<0$, then $I(u)<\min \{p+1, k+1\}(J(u)-d)$.

Proof (i) For $u \in N\left(\right.$ or $I(u)=0$ and $\left.\|u\|_{1, \Omega} \neq 0\right)$, by Lemma 2.3, we have $\|u\|_{1, \Omega}>\lambda_{0}$. Hence

$$
\begin{aligned}
J(u) & \geq \frac{1}{2}\|u\|_{1, \Omega}^{2}-\max \left\{\frac{1}{p+1}, \frac{1}{k+1}\right\}\left(\|u\|_{p+1}^{p+1}+\|u\|_{k+1, \Gamma}^{k+1}\right) \\
& =\left(\frac{1}{2}-\max \left\{\frac{1}{p+1}, \frac{1}{k+1}\right\}\right)\|u\|_{1, \Omega}^{2}+\max \left\{\frac{1}{p+1}, \frac{1}{k+1}\right\} I(u) \\
& =\left(\frac{1}{2}-\max \left\{\frac{1}{p+1}, \frac{1}{k+1}\right\}\right)\|u\|_{1, \Omega}^{2} \geq\left(\frac{1}{2}-\max \left\{\frac{1}{p+1}, \frac{1}{k+1}\right\}\right) \lambda_{0}^{2},
\end{aligned}
$$

which gives $d \geq d_{0}$. 
(ii) By Lemma 2.2, there exists $\lambda_{0} \in(0,1)$ such that $I\left(\lambda_{0} u\right)=0$ since $I(1 u)=I(u)<0$. Combining this $I\left(\lambda_{0} u\right)=0$ with the definition of $d$ and the fact that

$$
\begin{aligned}
J(u)= & \frac{1}{2}\|u\|_{1, \Omega}^{2}-\frac{1}{p+1}\|u\|_{p+1}^{p+1}-\frac{1}{k+1}\|u\|_{k+1, \Gamma}^{k+1} \\
= & \left(\frac{1}{2}-\max \left\{\frac{1}{p+1}, \frac{1}{k+1}\right\}\right)\|u\|_{1, \Omega}^{2}+\max \left\{\frac{1}{p+1}, \frac{1}{k+1}\right\} I(u) \\
& +\left[\max \left\{\frac{1}{p+1}, \frac{1}{k+1}\right\}-\frac{1}{p+1}\right]\|u\|_{p+1}^{p+1} \\
& +\left[\max \left\{\frac{1}{p+1}, \frac{1}{k+1}\right\}-\frac{1}{k+1}\right]\|u\|_{k+1, \Gamma}^{k+1},
\end{aligned}
$$

we obtain

$$
\begin{aligned}
d \leq & J\left(\lambda_{0} u\right) \\
= & \left(\frac{1}{2}-\max \left\{\frac{1}{p+1}, \frac{1}{k+1}\right\}\right) \lambda_{0}^{2}\|u\|_{1, \Omega}^{2} \\
& +\max \left\{\frac{1}{p+1}, \frac{1}{k+1}\right\} I\left(\lambda_{0} u\right)+\left[\max \left\{\frac{1}{p+1}, \frac{1}{k+1}\right\}-\frac{1}{p+1}\right] \lambda_{0}^{(p+1)}\|u\|_{p+1}^{p+1} \\
& +\left[\max \left\{\frac{1}{p+1}, \frac{1}{k+1}\right\}-\frac{1}{k+1}\right] \lambda_{0}^{(k+1)}\|u\|_{k+1, \Gamma}^{k+1} \\
\leq & \left(\frac{1}{2}-\max \left\{\frac{1}{p+1}, \frac{1}{k+1}\right\}\right)\|u\|_{1, \Omega}^{2}+\left[\max \left\{\frac{1}{p+1}, \frac{1}{k+1}\right\}-\frac{1}{p+1}\right]\|u\|_{p+1}^{p+1} \\
& +\left[\max \left\{\frac{1}{p+1}, \frac{1}{k+1}\right\}-\frac{1}{k+1}\right]\|u\|_{k+1, \Gamma}^{k+1} \\
= & J(u)-\max \left\{\frac{1}{p+1}, \frac{1}{k+1}\right\} I(u),
\end{aligned}
$$

which gives the result since $\max \left\{\frac{1}{p+1}, \frac{1}{k+1}\right\}=\min \{p+1, k+1\}$ for $p, k>1$.

Now we define the subsets of $H^{1}$ related to problem (1.1)-(1.3). Set

$$
W=\left\{u \in H^{1} \mid J(u)<d, I(u)>0\right\}, \quad V=\left\{u \in H^{1} \mid J(u)<d, I(u)<0\right\} .
$$

Lemma 2.5 If $u_{0}, u_{1} \in H^{1}$ and $0<E(0)<d, u$ is a weak solution of problem (1.1)-(1.3), then (i) $u \in W$ if $I\left(u_{0}\right)>0$ or $\|u\|_{1, \Omega}=0$; (ii) $u \in V$ if $I\left(u_{0}\right)<0$.

Proof We only prove (i), the proof for (ii) is similar. Let $T_{m}$ be maximal existence time of a weak solution of $u(t)$. We are going to prove that $u \in W$ for $0<t<T_{m}$. From the energy identity (2.5), we have

$$
E(t)=\frac{1}{2}\left\|u_{t}\right\|_{1, \Omega}^{2}+J(u)=E(0)<d \quad \text { for any } t \in[0, T)
$$

which implies $J(u(t))<d$. To prove that $u \in W$ for $0<t<T_{m}$, we argue by contradiction. Indeed, if it is not the case, there would exist $t_{0} \in\left(0, T_{m}\right)$ such that $u\left(t_{0}\right) \in N$. By the definition of $d=\inf _{u \in N} J(u)$, one has $d<J\left(u\left(t_{0}\right)\right)<E\left(t_{0}\right) \leq d$, we reach a contradiction. 


\section{Global existence and blow-up of solutions}

In this section, we prove the global existence and blow-up of solutions for problem (1.1)(1.3).

Theorem 3.1 Let $u_{0}, u_{1} \in H^{1}, 0<E(0)<d, I\left(u_{0}\right)>0$ or $\|u\|_{1, \Omega}=0$, and $p, k$ satisfy (2.1), then the weak solution $u$ of problem (1.1)-(1.3) in Theorem 2.1 can be extended to $(0, \infty)$.

Proof By Lemma 2.5, we have $u \in W$, then $I(u)>0$ and $J(u)<d$ for all $t \in(0, T)$. Therefore

$$
\begin{aligned}
& \left(\frac{1}{2}-\frac{1}{p+1}\right)\|u\|_{p+1}^{p+1}+\left(\frac{1}{2}-\frac{1}{k+1}\right)\|u\|_{k+1, \Gamma}^{k+1} \\
& \quad=\frac{1}{2}\left(\|u\|_{p+1}^{p+1}+\|u\|_{k+1, \Gamma}^{k+1}\right)-\frac{1}{p+1}\|u\|_{p+1}^{p+1}-\frac{1}{k+1}\|u\|_{k+1, \Gamma}^{k+1} \\
& \quad=\frac{1}{2}\|\nabla u\|^{2}-\frac{1}{2} I(u)-\frac{1}{p+1}\|u\|_{p+1}^{p+1}-\frac{1}{k+1}\|u\|_{k+1, \Gamma}^{k+1} \\
& \quad \leq J(u)<d
\end{aligned}
$$

for all $t \in(0, T)$. Define $\alpha=\min \left\{\frac{1}{2}-\frac{1}{p+1}, \frac{1}{2}-\frac{1}{k+1}\right\}>0$, then (3.1) implies

$$
\|u\|_{p+1}^{p+1}+\|u\|_{k+1, \Gamma}^{k+1}<\frac{d}{\alpha}
$$

for all $t \in(0, T)$. By the energy identity, the definition of $J(u)$ and (3.2), we have

$$
\begin{aligned}
\frac{1}{2}\left\|u_{t}\right\|^{2}+\frac{1}{2}\|u\|_{1, \Omega}^{2} & =E(0)+\frac{1}{p+1}\|u\|_{p+1}^{p+1}+\frac{1}{k+1}\|u\|_{k+1, \Gamma}^{k+1} \\
& <d\left(1+\frac{1}{\alpha} \max \left\{\frac{1}{p+1}, \frac{1}{k+1}\right\}\right)
\end{aligned}
$$

for all $t \in(0, T)$. It follows from (3.3) and from a standard continuous argument that the local weak solution $u$ furnished by Theorem 2.1 can be extended to the whole interval $[0, \infty)$, that is, $u$ is a global solution.

Theorem 3.2 Let $u_{0}, u_{1} \in H^{1}, 0<E(0)<d, I\left(u_{0}\right)<0$, and $p$, $k$ satisfy (2.1), then the weak solution $u$ of problem (1.1)-(1.3) blows up in finite time, that is, the maximum existence time $T_{m}$ of $u$ is finite and

$$
\lim _{t \rightarrow T_{m}} \sup \left[\|u\|_{1, \Omega}^{2}+\left\|u_{t}\right\|_{1, \Omega}^{2}\right]=+\infty
$$

Proof Arguing by contradiction, we assume that $T_{m}=+\infty$. Set

$$
H(t)=\|u\|_{1, \Omega}^{2}
$$

then by taking the time derivative of the function $H(t)$, performing integration by parts, and using equations (1.1) and (1.2), we get

$$
H^{\prime}(t)=2\left(u, u_{t}\right)+2\left(\nabla u, \nabla u_{t}\right)
$$




$$
\begin{aligned}
H^{\prime \prime}(t) & =2\left\|u_{t}\right\|^{2}+2\left\|\nabla u_{t}\right\|^{2}+2\left(u, u_{t t}\right)+2\left(\nabla u, \nabla u_{t t}\right) \\
& =2\left\|u_{t}\right\|^{2}+2\left\|\nabla u_{t}\right\|^{2}-2 I(u) .
\end{aligned}
$$

By virtue of the Schwarz inequality, we have

$$
H^{\prime 2}(t) \leq 4 H(t)\left(\left\|u_{t}\right\|^{2}+\left\|\nabla u_{t}\right\|^{2}\right)
$$

Hence

$$
\begin{aligned}
H(t) H^{\prime \prime}(t)-\frac{\rho+2}{4} H^{\prime 2}(t) & \geq H(t)\left[H^{\prime \prime}(t)-(\rho+2)\left(\left\|u_{t}\right\|^{2}+\left\|\nabla u_{t}\right\|^{2}\right)\right] \\
& =H(t)\left[-\rho\left(\left\|u_{t}\right\|^{2}+\left\|\nabla u_{t}\right\|^{2}\right)-2 I(u)\right],
\end{aligned}
$$

where we denote $\rho=\min \{k, p\}$. Next, we treat the part $-\rho\left(\left\|u_{t}\right\|^{2}+\left\|\nabla u_{t}\right\|^{2}\right)$ in the above estimate. By the energy identity $E(t)=E(0)$, we have

$$
-\rho\left(\left\|u_{t}\right\|^{2}+\left\|\nabla u_{t}\right\|^{2}\right)=2 \rho(J(u)-E(0)) .
$$

Substituting this into (3.7), we find

$$
\begin{aligned}
H(t) H^{\prime \prime}(t)-\frac{\rho+2}{4} H^{\prime 2}(t) & \geq 2 H(t)[\rho(J(u)-E(0))-I(u)] \\
& \geq 2 H(t)[\rho(J(u)-d))-I(u)] .
\end{aligned}
$$

From $u_{0} \in V$ and Lemma 2.5, we have $u(t) \in V$, that is, $I(u)<0$ for all $0<t<\infty$, then Lemma 2.4(ii) holds, by (3.8), which leads to

$$
H(t) H^{\prime \prime}(t)-\frac{\rho+2}{4} H^{\prime 2}(t) \geq 0
$$

So,

$$
\left(H^{-\beta}(t)\right)^{\prime \prime}=\frac{-\beta}{H^{\beta+2}(t)}\left(H(t) H^{\prime \prime}(t)-(\beta+1) H^{\prime 2}(t)\right)<0, \quad \beta=\frac{\rho}{4} .
$$

From (3.6), Lemma 2.4(ii), and $E(0)<d$, we see that

$$
H^{\prime \prime}(t) \geq-2 I(u) \geq 2 \rho(d-J(u)) \geq 2 \rho(d-E(0)),
$$

and then

$$
H^{\prime}(t) \geq 2 \rho(d-E(0))+H^{\prime}(0)
$$

Hence there exists $t_{0} \geq 0$ such that $H^{\prime}(t)>H^{\prime}(0)>0$ for $t_{0}<+\infty$ and

$$
H(t)>H^{\prime}(0)\left(t-t_{0}\right)+H\left(t_{0}\right) \geq H^{\prime}(0)\left(t-t_{0}\right) .
$$

Consequently, there exists $t_{1}$ such that $H\left(t_{1}\right)>0$ and $H^{\prime}\left(t_{1}\right)>0$. From this and (3.9), one can find $T_{1}>0$ such that $\lim _{t \rightarrow T_{1}} H^{-\beta}(t)=0$, therefore $\lim _{t \rightarrow T_{1}} H(t)=+\infty$, which contradicts $T_{m}=+\infty$. Finally, from $T_{m}<+\infty$ and Theorem 2.1 , we get the result. 
Remark Combining this method with the method of $[18,19,27]$, we can also consider equations (1.7), (1.8).

\author{
Acknowledgements \\ The authors would like to thank the referees for their useful suggestions. \\ Funding \\ This work is supported by the National Natural Science Foundation of China (No. 11801145).
}

Availability of data and materials

Not applicable.

Competing interests

The authors declare that they have no competing interests.

\title{
Authors' contributions
}

The authors declare that the study was realized in collaboration with the same responsibility. All authors read and approved the final manuscript.

\section{Publisher's Note}

Springer Nature remains neutral with regard to jurisdictional claims in published maps and institutional affiliations.

\section{Received: 8 October 2018 Accepted: 15 January 2019 Published online: 23 January 2019}

\section{References}

1. Korpusov, M.O.: Blow-up of ion-sound waves in plasma with non-linear sources on the boundary. Izv. Math. 76(2), 310-345 (2012)

2. Gabov, S.A.: New Problems of the Mathematical Theory of Waves. Fizmatlit, Moscow (1998) (in Russian)

3. Al'shin, A.B., Pletner, Y.D., Sveshnikov, A.G.: Unique solvability of the Dirichlet problem for the ion-sound wave equation in plasma. Dokl. Math. 58(1), 148-150 (1998)

4. Alghamdi, A.M., Gala, S., Ragusa, M.A.: On the blow-up criterion for incompressible Stokes-MHD equations. Results Math. 73(3), Article number 110 (2014)

5. Gala, S., Ragusa, M.A., Sawano, Y., Tanaka, H.: Uniqueness criterion of weak solutions for the dissipative quasi-geostrophic equations in Orlicz-Morrey spaces. Appl. Anal. 93(2), 356-368 (2014)

6. Gala, S., Guo, Z., Ragusa, M.A.: A remark on the regularity criterion of Boussinesq equations with zero heat conductivity. Appl. Math. Lett. 27, 70-73 (2014)

7. Levine, H.A.: Instability and nonexistence of global solutions to nonlinear wave equations of the form $P u_{t t}=-A u+F(u)$. Trans. Am. Math. Soc. 192, 1-21 (1974)

8. Kalantarov, V.K., Ladyzhenskaya, O.A.: The occurrence of collapse for quasilinear equations of parabolic and hyperbolic types. J. Math. Sci. 10(1), 53-70 (1978)

9. Park, J.Y., Kim, J.A.: Existence and uniform decay for a nonlinear viscoelastic equation with strong damping and nonlinear boundary memory damping term. Dyn. Syst. Appl. 18, 605-620 (2009)

10. Sakamoto, R.: Mixed problems for hyperbolic equations. J. Math. Kyoto Univ. 10(2), 243-373 (1970)

11. Bociu, L., Rammaha, M., Toundykov, D.: Wave equations with super-critical interior and boundary nonlinearities. Math. Comput. Simul. 82, 1017-1029 (2012)

12. Lasiecka, I., Tataru, D.: Uniform boundary stabilization of semilinear wave equations with nonlinear boundary damping. Differ. Integral Equ. 6(3), 507-533 (1993)

13. Vitillaro, E.: Global existence for the wave equation with nonlinear boundary damping and source terms. J. Differ. Equ. 186(1), 259-298 (2002)

14. Bociu, L.: Local and global well posedness of weak solutions for the wave equation with nonlinear boundary and interior sources of supercritical exponents and damping. Nonlinear Anal. 71(12), e560-e575 (2009)

15. Bociu, L., Lasiecka, I.: Blow-up of weak solutions for the semilinear wave equations with nonlinear boundary and interior sources and damping. Appl. Math. 35, 281-304 (2008)

16. Bociu, L., Lasiecka, I.: Uniqueness of weak solutions for the semilinear wave equations with supercritical boundary/interior sources and damping. Discrete Contin. Dyn. Syst. 22, 835-860 (2008)

17. Bociu, L., Lasiecka, I.: Hadamard well posedness for nonlinear wave equations with supercritical sources and damping. J. Differ. Equ. 249, 654-683 (2010)

18. Bociu, L., Rammaha, M., Toundykov, D.: On a wave equation with supercritical interior and boundary sources and damping terms. Math. Nachr. 284(16), 2032-2064 (2011)

19. Cavalcanti, M.M., Cavalcanti, V.N.D., Lasiecka, I.: Well-posedness and optimal decay rates for wave equation with nonlinear boundary damping-source interaction. J. Differ. Equ. 236, 407-459 (2007)

20. Cavalcanti, M.M., Cavalcanti, V.N.D., Martinez, P.: Existence and decay rates for the wave equation with nonlinear boundary damping and source term. J. Differ. Equ. 203, 119-158 (2004)

21. Cavalcanti, M.M., Cavalcanti, V.N.D., Soriano, J.: Global solvability and asymptotic stability for the wave equation with nonlinear feedback and source term on the boundary. Adv. Math. Sci. Appl. 16, 661-696 (2006)

22. Chueshov, I., Eller, M., Lasiecka, I.: On the attractor for a semilinear wave equation with critical exponent and nonlinear boundary dissipation. Commun. Partial Differ. Equ. 27, 1901-1951 (2002)

23. Daoulatli, M., Lasiecka, I., Toundykov, D.: Uniform energy decay for a wave equation with partially supported nonlinear boundary dissipation without growth restrictions. Discrete Contin. Dyn. Syst., Ser. S 2, 67-94 (2009) 
24. Guo, Y.Q., Rammaha, M.A.: Global existence and decay of energy to systems of wave equations with damping and supercritical sources. Z. Angew. Math. Phys. 64, 621-658 (2013)

25. Guo, Y.Q., Rammaha, M.A.: Systems of nonlinear wave equations with damping and supercritical boundary and interior sources. Trans. Am. Math. Soc. 366(5), 2265-2325 (2014)

26. Levine, H., Smith, A.: A potential well theory for the wave equation with a nonlinear boundary conditions. J. Reine Angew. Math. 374, 1-23 (1987)

27. Wu, S.T.: General decay and blow-up of solutions for a viscoelastic equation with nonlinear boundary damping-source interactions. Z. Angew. Math. Phys. 63, 65-106 (2012)

28. Wu, S.T.: Blow-up of positive initial energy solutions for a system of nonlinear wave equations with supercritical sources. J. Dyn. Control Syst. 20(2), 207-227 (2014)

29. Ji, G., Lasiecka, I.: Nonlinear boundary feedback stabilization for a semilinear Kirchhoff plate with dissipation acting only via moments-limiting behavior. J. Math. Anal. Appl. 229(2), 452-479 (1999)

30. Lions, J.L:: Quelques methodes de resolution des problemes aur limites non lineaires. Dunod/Gauthier-Villars, Paris (1969)

Submit your manuscript to a SpringerOpen ${ }^{0}$ journal and benefit from:

- Convenient online submission

- Rigorous peer review

Open access: articles freely available online

- High visibility within the field

- Retaining the copyright to your article

Submit your next manuscript at $\boldsymbol{\nabla}$ springeropen.com 